Pacific Journal of Mathematics

SOME APPLICATIONS OF MEANS OF CONVEX BODIES 


\section{SOME APPLICATIONS OF MEANS OF CONVEX BODIES}

\section{WilLiAM J. FiREY}

Let $A$ be a real, positive definite, $n \times n$ matrix; with $A$ we associate, in the Euclidean $n$-space $R_{n}$, the ellipsoid $E(A)$ of points $x$ for which

$$
(x, A x) \leqq 1
$$

where $(x, y)$ denotes the usual inner product. In references [5], [6], [7] certain means of convex bodies were studied. It will be shown here that two particular means of ellipsoids of the type $E(A)$ correspond to two simple combinations of the corresponding matrices $A$. The applications mentioned in the title rest upon this correspondence. The first two give results about positive definite matrices, including a refinement of a determinant inequality of Minkowski; the third application shows the existence of a set of unique ellipsoids related to a convex body by a set of similar extremal problems, the classical Loewner ellipsoid being a particular instance.

Throughout this paper the letters $A$ and $B$, sometimes with distinguishing marks, denote real, positive definite, $n \times n$ matrices. The distance from $x$ to the origin is written $\|x\|$.

1. The distance and support functions of $E(A)$ are:

$$
F(x)=\sqrt{ }(x, A x), \quad H(x)=\sqrt{ }\left(x, A^{-1} x\right) .
$$

In the first case, if $x \neq 0$, we have $F(x)=\|x\| /\|z\|$ where $x /\|x\|=$ $z /\|z\|$ and $(z, A z)=1$, and so

$$
\begin{aligned}
\|x\| /\|z\| & =\|x\| V(z /\|z\|, A z /\|z\|) \\
& =\|x\| \sqrt{ }(x /\|x\|, A x /\|x\|)=V(x, A x) .
\end{aligned}
$$

In the second case

$$
H(x)=\max _{y}(x, y) \quad \text { where } \quad(y, A y)=1 .
$$

We represent $y$ in the form $\lambda A^{-1} x+v$ where $(x, v)=0$. Then

$$
(y, A y)=\lambda^{2}\left(x, A^{-1} x\right)+(v, A v),
$$

whence

Received April 4, 1963. This work was supported in part by a grant from the National Science Foundation, NSF-G19838. The author is grateful to the referee for a constructive comment on the last application. 


$$
(x, y)=\lambda\left(x, A^{-1} x\right)=\sqrt{ }\left(x, A^{-1} x\right) \bigvee[1-(v, A v)],
$$

and the maximum is attained for $v=0$.

The polar reciprocal $\hat{E}(A)$ of $E(A)$ with respect to the unit sphere $E(I)$ has $H(x)$ as its distance function, $F(x)$ as its support function. Consequently

$$
\widehat{E}(A)=E\left(A^{-1}\right) .
$$

In [5] the $p$-dot mean of two convex bodies $K_{0}, K_{1}$ in $R_{n}$, which have the origin as a common interior point, was defined for $p \geqq 1$ to. be to convex body $\dot{M}_{p}\left(K_{0}, K_{1} ; \vartheta\right)$ whose distance function is

$$
\left[(1-\vartheta) F_{0}^{p}(x)+\vartheta F_{1}^{p}(x)\right]^{1 / p}
$$

where $F_{i}$ is the distance function of $K_{i}$ and $0 \leqq \vartheta \leqq 1$. From this it. follows that $\dot{M}_{2}\left(E\left(A_{0}\right), E\left(A_{1}\right) ; \vartheta\right)$ has the distance function

$$
\checkmark\left[(1-\vartheta)\left(x, A_{0} x\right)+\vartheta\left(x, A_{1} x\right)\right]=V\left(x,\left[(1-\vartheta) A_{0}+\vartheta A_{1}\right] x\right) .
$$

Thus

$$
\dot{M}_{2}\left(E\left(A_{0}\right), E\left(A_{1}\right) ; \vartheta\right)=E\left((1-\vartheta) A_{0}+\vartheta A_{1}\right) .
$$

In [7] the $p$-mean $M_{p}\left(K_{0}, K_{1} ; \vartheta\right)$ was defined for $p \geqq 1$ to be the convex body whose support function is

$$
\left[(1-\vartheta) H_{0}^{p}(x)+\vartheta H_{1}^{p}(x)\right]^{1 / p}
$$

where $H_{i}$ is the support function of $K_{i}$. Therefore, by reasoningsimilar to the preceding, we have

$$
M_{2}\left(E\left(A_{0}\right), E\left(A_{1}\right) ; \vartheta\right)=E\left(\left[(1-\vartheta) A_{0}^{-1}+\vartheta A_{1}^{-1}\right]^{-1}\right) .
$$

2. Our first application is based on the inclusion

$$
\dot{M}_{2}\left(K_{0}, K_{1} ; \vartheta\right) \subseteq M_{2}\left(K_{0}, K_{1} ; \vartheta\right),
$$

established in [5] and [7] $]^{1}$ with equality if and only if $K_{0}=K_{1}$, and the observation that

$$
E(A) \subseteq E(B)
$$

if and only if $A-B$ is positive semi-definite. For the latter we write $A \geqq B$; we call such an inequality strict if $A-B$ is not a zero matrix. From (2), (3) and (4) we have

$$
E\left((1-\vartheta) A_{0}+\vartheta A_{1}\right) \subseteq E\left(\left[(1-\vartheta) A_{0}^{-1}+\vartheta A_{1}^{-1}\right]^{-1}\right) .
$$

1 The inclusion is not specifically mentioned, but in [7] it is proved that $M_{1} \subseteq M_{p}$ for $p>1$ and in [5] that $\dot{M}_{p} \cong \dot{M}_{1}$ and $\dot{M}_{1} \subseteq M_{1}$. 
Hence, from (5) we obtain an "inequality of arithmetic and harmonic means" for positive definite matrices.

THEOREm 1. If $A_{0}, A_{1}$ are any two real, positive definite, $n \times n$ matrices, then

$$
(1-\vartheta) A_{0}+\vartheta A_{1} \geqq\left[(1-\vartheta) A_{0}^{-1}+\vartheta A_{1}^{-1}\right]^{-1}
$$

for $0 \leqq \vartheta \leqq 1$. The inequality is strict except in the trivial cases $A_{0}=A_{1}$ or $\vartheta=0,1$.

3. The next application is a refinement of the following determinant inequality of Minkowski, ef [1], p. 70.

$$
\operatorname{det}^{1 / n}\left(A_{0}+A_{1}\right) \geqq \operatorname{det}^{1 / n} A_{0}+\operatorname{det}^{1 / n} A_{1} .
$$

Let $V$ be the volume functional. In [5] it was shown that

$$
V\left(\dot{M}_{p}\left(K_{0}, K_{1} ; \vartheta\right)\right) \leqq\left[(1-\vartheta) V^{-p / n}\left(K_{0}\right)+\vartheta V^{-p / n}\left(K_{1}\right)\right]^{-n / p}
$$

with equality if and only if $K_{0}=\lambda K_{1}$ for some $\lambda>0$. Since

$$
V(E(A))=\pi^{n / 2} / \Gamma(1+n / 2) \vee \operatorname{det} A,
$$

we have, with $p=2$ in (6),

$$
\operatorname{det}\left[(1-\vartheta) A_{0}+\vartheta A_{1}\right] \geqq\left[(1-\vartheta) \operatorname{det}^{1 / n} A_{0}+\vartheta \operatorname{det}^{1 / n} A_{1}\right]^{n}
$$

with equality if and only if $A_{0}=\lambda A_{1}$ for some $\lambda>0$. With a slight change in notation, this is Minkowski's determinant inequality.

If $L$ is any $k$-dimensional linear subspace of $R_{n}$, then

$$
\dot{M}_{2}\left(E\left(A_{0}\right) \cap L, E\left(A_{1}\right) \cap L ; \vartheta\right)=\dot{M}_{2}\left(E\left(A_{0}\right), E\left(A_{1}\right) ; \vartheta\right) \cap L .
$$

Consequently, by letting $A^{\prime}$ be the $k \times k$, positive definite matrix associated with $E(A) \cap L$, we obtain

$$
E\left((1-\vartheta) A_{0}^{\prime}+\vartheta A_{1}^{\prime}\right)=E\left(\left[(1-\vartheta) A_{0}+\vartheta A_{1}\right]^{\prime}\right) .
$$

To this we apply (7), with $n=k$, to get

$$
\operatorname{det}\left[(1-\vartheta) A_{0}+\vartheta A_{1}\right]^{\prime} \geqq\left[(1-\vartheta) \operatorname{det}^{1 / k} A_{0}^{\prime}+\vartheta \operatorname{det}^{1 / k} A_{1}^{\prime}\right]^{k} .
$$

Let us define $|A|_{k}$ to be the product of the $k$ least eigenvalues of $A$, repeated eigenvalues being counted according to their multiplicity. The inequality

$$
\operatorname{det} A^{\prime} \geqq|A|_{k}
$$

with equality if and only if $L$ is the $k$-dimensional space spanned by the eigenvectors corresponding to the $k$ least eigenvalues of $A$, is 
essentially Theorem 20 , p. 74 of [1].

In (8) choose $L$ to be the linear subspace spanned by those eigenvectors of $(1-\vartheta) A_{0}+\vartheta A$, which correspond to the $k$ smallest eigenvalues of $(1-\vartheta) A_{0}+\vartheta A_{1}$. By (9):

$$
\operatorname{det} A_{0}^{\prime} \geqq\left|A_{0}\right|_{k}, \quad \operatorname{det} A_{1}^{\prime} \geqq\left|A_{1}\right|_{k},
$$

and so (8) becomes

$$
\left|(1-\vartheta) A_{0}+\vartheta A_{1}\right|_{k} \geqq\left[(1-\vartheta)\left|A_{0}\right|_{k}^{1 / / k}+\vartheta\left|A_{1}\right|_{k}^{1 / k}\right]^{k} .
$$

There is equality in (8) if and only if, for some $\lambda>0$,

$$
A_{0}^{\prime}=\lambda A_{1}^{\prime}
$$

and equality in (10) if and only if the subspaces $L$ appropriate to $\left|A_{0}\right|_{k},\left|A_{1}\right|_{k}$ are the same. Hence, in (11), there is equality if and only if the following conditions are met. Let $x_{1}, \cdots, x_{k}$ be eigenvectors of $A_{0}$ corresponding to the $k$ smallest eigenvalues $\lambda_{1} \leqq \cdots \leqq \lambda_{k}$. These are eigenvectors of $A_{1}$ corresponding to the $k$ smallest eigenvalues of $A_{1}$ which are of the form $\lambda \lambda_{1} \leqq \cdots \leqq \lambda \lambda_{k}$ for some $\lambda>0$.

Inequality (11), which includes (7) when $k=n$, is an improvement of a result of Ky Fan, cf. [1], Theorem 21, p. 74, in which the right side of (11) is replaced by the geometric mean $\left|A_{0}\right|_{k}^{1-9}\left|A_{1}\right|_{k}^{9}$ since the power mean of order $1 / k$ appearing on the right side of (11) exceeds this geometric mean.

If we define ${ }_{k}|A|$ to be the product of the $k$ greatest eigenvalues of $A$, then

$$
\left|A^{-1}\right|_{k}=1 /{ }_{k}|A| \text {. }
$$

We apply (11) to $(1-\vartheta) A_{0}^{-1}+\vartheta A_{1}^{-1}$ and obtain, after taking reciprocals,

$$
1 /\left|(1-\vartheta) A_{0}^{-1}+\vartheta A_{1}^{-1}\right|_{k} \leqq\left[(1-\vartheta)_{k}\left|A_{0}\right|^{-1 / k}+\vartheta_{k}\left|A_{1}\right|^{-1 / k}\right]^{-k}
$$

With the use of (12) on the left side, we have finally

$$
{ }_{k}\left|\left[(1-\vartheta) A_{0}^{-1}+\vartheta A_{1}^{-1}\right]^{-1}\right| \leqq\left[(1-\vartheta)_{k}\left|A_{0}\right|^{-1 / k}+\vartheta_{k}\left|A_{1}\right|^{-1 / k}\right]^{-k}
$$

as a "dual" result to (11). The cases of equality are given by the conditions for equality in (11) with the word "smallest" replaced by "greatest" throughout.

The last application concerns a generalization of the Loewner ellipsoid of a convex body $K$. Let $x$ be an interior point of $K$. The classical Loewner ellipsoid is that unique ellipsoid, centred at $x$ and containing $K$, which has minimum volume, cf. [3]. Let us take the point $x$ to be the origin and denote the mean cross-sectional measures $W_{\nu}, \nu=0,1, \cdots, n-1$, of $E(A)$ by $W_{\nu}(A)$; for their definition see 
[2]. In particular $W_{0}(A)=V(E(A))$. We will show that, for each $\nu$ there is a unique ellipsoid $E(A)$ containing $K$ for which $W_{\nu}(A)$ is a minimum.

It is clear that $W_{\nu}(A)$ depends continuously on the entries $a_{i j}$ of $A$. Moreover, when we restrict the ellipsoids $E(A)$ not only to contain $K$, but also to be contained in the sphere $E\left(I / \rho^{2}\right)$, the domain of definition of the functions $W_{\nu}(A)$ is closed and bounded. Consequently each of the functions $W_{\nu}(A)$ attains a minimum. Furthermore, if the radius of the bounding sphere $E\left(I / \rho^{2}\right)$ is chosen to be sufficiently large, the minimum of $W_{\nu}(A)$ and the matrix or matrices for which it is attained will be independent of $\rho$. Thus the uniqueness is the only point in question.

In [6] inequality (6) was extended to read

$$
W_{\nu}^{1 /(n-\nu)}\left[\dot{M}_{p}\left(K_{0}, K_{1} ; \vartheta\right)\right] \leqq\left[(1-\vartheta) W_{\nu}^{-p /(n-\nu)}\left(K_{0}\right)+\vartheta W_{\nu}^{-p /(n-\nu)}\left(K_{1}\right)\right]^{-1 / p}
$$

for $p=1$, with equality if and only if $K_{0}=\lambda K_{1}$ for some $\lambda>0$. Inequality (13) is true for all $p \geqq 1$ however. This can be shown from the special case $p=1$ in the following fashion. We make the usual type of reduction to the special case in which $W_{\nu}\left(K_{i}\right)=1, i=0,1$, by setting:

$$
\begin{aligned}
& \lambda_{i}=W_{\nu}^{1 /(n-\nu)}\left(K_{i}\right), \quad K_{i}=\lambda_{i} K_{i}^{\prime}, \\
& \vartheta^{\prime}=\vartheta \lambda_{1}^{-p} /\left[(1-\vartheta) \lambda_{0}^{-p}+\vartheta \lambda_{1}^{-p}\right] .
\end{aligned}
$$

Then

$$
\dot{M}_{p}\left(K_{0}^{\prime}, K_{1}^{\prime} ; \vartheta^{\prime}\right)=\dot{M}_{p}\left(K_{0}, K_{1} ; \vartheta\right) / \mu
$$

where

$$
\mu=\left[(1-\vartheta) \lambda_{0}^{-p}+\vartheta \lambda_{1}^{-p}\right]^{-1 / p} .
$$

Since $W_{\nu}\left(K_{i}^{\prime}\right)=1$, in order to prove (13) it is enough to prove

$$
W_{\nu}\left(\dot{M}_{p}\left(K_{0}^{\prime}, K_{1}^{\prime} ; \vartheta^{\prime}\right) \leqq 1\right. \text {. }
$$

This has been shown to be true for $p=1$. By Theorem 2 of [5]

$$
\dot{M}_{p}\left(K_{0}^{\prime}, K_{1}^{\prime} ; \vartheta^{\prime}\right) \subseteq \dot{M}_{1}\left(K_{0}^{\prime}, K_{1}^{\prime} ; \vartheta^{\prime}\right)
$$

with equality if and only if $K_{0}^{\prime}=K_{1}^{\prime}$. These assertions, together with the monotonic character of $W_{\nu}$ cf. [2], p. 50, prove (13) and establish the cases of equality. Naturally we will use (13) for $p=2$.

Let $A_{\nu}$ be a matrix which is a solution of the minimum problem:

$$
K \leqq E(A), \quad W_{\nu}(A)=\text { minimum. }
$$

Suppose $A_{\nu}^{\prime}$ is a second solution. From 


$$
K \subseteq E\left(A_{\nu}\right), \quad K \subseteq E\left(A_{\nu}^{\prime}\right)
$$

we have

$$
K \subseteq E\left((1-\vartheta) A_{\nu}+\vartheta A_{\nu}^{\prime}\right)
$$

from (13) we have

$$
W_{\nu}\left((1-\vartheta) A_{\nu}+\vartheta A_{\nu}^{\prime}\right) \leqq W_{\nu}\left(A_{\nu}\right)=W_{\nu}\left(A_{\nu}^{\prime}\right)
$$

with equality in the inequality if and only if $A_{\nu}=\lambda A_{\nu}^{\prime}$. The last equality shows that we must have $\lambda=1$ and so $A_{\nu}$ is unique.

In a similar way we can establish that, given $K$ and an interior point of $K$ which we take as the origin, there is a unique ellipsoid $E\left(B_{\nu}\right)$ which is contained in $K$ for which is a maximum. The only difference is the use of Theorem 2 of [7] in lieu of inequality (13).

We summarize:

Theorem 2. Given a convex body $K$ in Euclidean $n$-space and an interior point of $K$ which we take as the origin, there are positive definite $n \times n$ matrices $A_{\nu}, B_{\nu}, \nu=0,1, \cdots, n-1$ such that, among the ellipsoids $E(A)$ which contain $K, E\left(A_{\nu}\right)$ is the unique, outer, Loewner ellipsoid minimizing $W_{\nu}$ and among the ellipsoids $E(B)$ which are contained in $K, E\left(B_{\nu}\right)$ is the unique inner, Loewner ellipsoid maximizing $W_{\nu}$.

We close with several observations. Suppose $\hat{K}$ is the polar reciprocal of $K$ with respect to $E(I)$, then, in the notation of Theorem $2, E\left(B_{\nu}^{-1}\right)$ is the $\nu$ th outer Loewner ellipsoid of $\hat{K}$ while $E\left(A_{\nu}^{-1}\right)$ is the $\nu$ th inner Loewner ellipsoid. To prove this, we denote the outer and inner Loewner ellipsoids of $\hat{K}$ with respect to the origin by $E\left(\hat{A}_{\nu}\right)$, $E\left(\hat{B}_{\nu}\right)$ respectively. If $K_{0} \subseteq K_{1}$, then $\hat{K}_{0} \supseteqq \hat{K}_{1}$. Consequently, by (1),

$$
\hat{E}\left(A_{\nu}\right)=E\left(A_{\nu}^{-1}\right) \leqq \hat{K}, \quad \hat{E}\left(B_{\nu}\right)=E\left(B_{\nu}^{-1}\right) \supseteqq \hat{K} .
$$

Therefore

$$
E\left(A_{\nu}^{-1}\right) \cong E\left(\hat{B}_{\nu}\right), \quad E\left(B_{\nu}^{-1}\right) \supseteqq E\left(\hat{A}_{\nu}\right) .
$$

Applying the same argument to $\hat{A}_{\nu}$ and $\hat{B}_{\nu}$ we get

$$
E\left(\hat{A}_{\nu}^{-1}\right) \subseteq E\left(B_{\nu}\right), \quad E\left(\hat{B}_{\nu}^{-1}\right) \supseteqq E\left(A_{\nu}\right) .
$$

In terms of the ordering of positive definite matrices, these inclusions become

$$
A_{\nu}^{-1} \geqq \hat{B}_{\nu}, \quad \hat{A}_{\nu} \geqq B_{\nu}^{-1}, \quad \hat{A}_{\nu}^{-1} \geqq B_{\nu}, \quad A_{\nu} \geqq \hat{B}_{\nu}^{-1} .
$$


Now when $B \geqq A$, then $A^{-1} \geqq B^{-1}$ since, from the first condition we have

$$
E(A) \supseteqq E(B)
$$

and, by taking polar reciprocals, we obtain

$$
E\left(A^{-1}\right) \subseteq E\left(B^{-1}\right) \text {. }
$$

Apply this to the last inequality of (14). Taken together with the first inequality of (14), this yields

$$
A^{-1} \geqq \hat{B}_{\nu} \geqq A_{\nu}^{-1} \text {. }
$$

Thus $\hat{B}_{\nu}-A_{\nu}^{-1}$ is both positive and negative semi-definite. Hence

$$
A_{\nu}^{-1}=\hat{B}_{\nu} \text {. }
$$

By a similar argument it is shown that

$$
B_{\nu}^{-1}=\hat{A}_{\nu} \text {. }
$$

Part of Theorem 2 remains true even if the centre of the ellipsoids to be considered does not lie within $K$. We give this as a corollary.

Corollary to Theorem 2. Given a convex body $K$, not necessarily containing the origin, there are positive definite matrices $A_{\nu}, \nu=$ $0,1, \cdots, n-1$, such that, among the ellipsoids $E(A)$ which contain $K, E\left(A_{\nu}\right)$ is the unique outer Loewner ellipsoid minimizing $W_{\nu}$.

Suppose $E(A)$ contains $K$; since $E(A)$ is centred at the origin it also contains a sufficiently small sphere $E(\rho I)$ and so, by the convexity of $E(A), E(A)$ contains

$$
K^{\prime}=\overline{K \cup E(\rho I)}
$$

where the bar denotes the convex closure. Conversely, if $E(A)$ contains $K^{\prime}$ it contains the subset $K$. We claim as proof of the corollary that the outer Loewner ellipsoid $E\left(A_{v}\right)$ of $K^{\prime}$ is also that of $K$. Indeed $E\left(A_{\nu}\right)$ contains $K$ and if an ellipsoid $E\left(A_{\nu}^{\prime}\right)$ contains $K$ and is such that

$$
W_{\nu}\left(A_{\nu}^{\prime}\right) \leqq W_{\nu}\left(A_{\nu}\right)
$$

then $E\left(A_{\imath}^{\prime}\right)$ must contain $K^{\prime}$ and so, by Theorem 2, $A^{\prime}=A_{\nu}$.

Let $x$ be the interior point mentioned in Theorem 2 and let $E\left(A_{\nu}(x)\right)$, $E\left(B_{\nu}(x)\right)$ be the $\nu$ th outer and inner Loewner ellipsoids of $K$ which are centred at $x$. We allow $x$ to vary and so generate two collections of ellipsoids $\left\{E\left(A_{\nu}(x)\right)\right\}$ and $\left\{E\left(B_{\nu}(x)\right)\right\}$. For $\nu=0$ Danzer, Laugwitz and Lenz in [4] have shown that in the first collection there is a unique 
ellipsoid for which the volume $W_{0}$ is a minimum and in the second collection there is a unique ellipsoid for which the volume is a maximum. We have not been able to decide if this is also true for $\nu=1$, $2, \cdots, n-1$ with $W_{\nu}$ in place of the volume.

\section{REFERENCES}

1. E. Beckenbach and R. Bellman, Inequalities, Berlin, Göttingen, Heidelberg, 1961.

2. T. Bonnesen and W. Fenchel, Theorie der konvexen Körper, Berlin, 1934

3. H. Busemann, The Geometry of Geodesics, New York, 1955.

4. L. Danzer, D. Laugwitz and H. Lenz, Über das Löwnersche Ellipsoid und sein Analogen unter den Eikörper einbeschriebenen Ellipsoiden, Archiv der Mathematik, 8 (1957), 214-218.

5. W. Firey, Polar means of convex bodies and a dual to the Brunn-Minkowski theorem, Canadian J. Math., 13 (1961) 444-453.

6 . Mean cross-section measures of harmonic means of convex bodies, Pacific J. Math., 11 (1961), 1263-1266.

7. —

OREgon STATE UNIVERSITY 


\section{PACIFIC JOURNAL OF MATHEMATICS}

\section{EDITORS}

Robert Osserman

Stanford University

Stanford, California

M. G. Arsove

University of Washington

Seattle 5 , Washington
J. DugundjI

University of Southern Califorma: Los Angeles 7, California

Lowell J. Paige

University of California

Los Angeles 24, California

\section{ASSOCIATE EDITORS}

E. F. BECKENBACH

B. H. NeumanN

F. WOLF

K. YosIDA

\section{SUPPORTING INSTITUTIONS}

UNIVERSITY OF BRITISH COLUMBIA

CALIFORNIA INSTITUTE OF TECHNOLOGY

UNIVERSITY OF CALIFORNIA

MONTANA STATE UNIVERSITY

UNIVERSITY OF NEVADA

NEW MEXICO STATE UNIVERSITY

OREGON STATE UNIVERSITY

UNIVERSITY OF OREGON

OSAKA UNIVERSITY

UNIVERSITY OF SOUTHERN CALIFORNIA
STANFORD UNIVERSITY

UNIVERSITY OF TOKYO

UNIVERSITY OF UTAH

WASHINGTON STATE UNIVERSITY

UNIVERSITY OF WASHINGTON

AMERICAN MATHEMATICAL SOCIETY CALIFORNIA RESEARCH CORPORATION SPACE TECHNOLOGY LABORATORIES NAVAL ORDNANCE TEST STATION 


\section{Pacific Journal of Mathematics}

\section{Vol. 14, No. 1 \\ May, 1964}

Richard Arens, Normal form for a Pfaffian .........................

Charles Vernon Coffman, Non-linear differential equations on cones in Banach

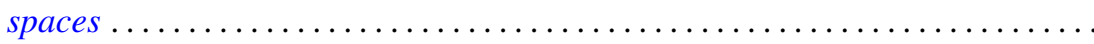

Ralph DeMarr, Order convergence in linear topological spaces ..............

Peter Larkin Duren, On the spectrum of a Toeplitz operator ................

Robert E. Edwards, Endomorphisms of function-spaces which leave stable all

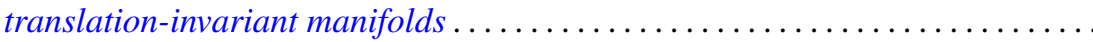

Erik Maurice Ellentuck, Infinite products of isols . . . . . . . . . . . . . . . . 49

William James Firey, Some applications of means of convex bodies . . . . . . . . 53

Haim Gaifman, Concerning measures on Boolean algebras ............. 61

Richard Carl Gilbert, Extremal spectral functions of a symmetric operator. . . . . . 75

Ronald Lewis Graham, On finite sums of reciprocals of distinct nth powers ..... 85

Hwa Suk Hahn, On the relative growth of differences of partition functions ...... 93

Isidore Isaac Hirschman, Jr., Extreme eigen values of Toeplitz forms associated

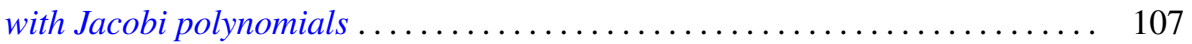

Chen-jung Hsu, Remarks on certain almost product spaces . . . . . . . . . . . 163

George Seth Innis, Jr., Some reproducing kernels for the unit disk . . . . . . . . . 177

Ronald Jacobowitz, Multiplicativity of the local Hilbert symbol . . . . . . . . . . . 187

Paul Joseph Kelly, On some mappings related to graphs ................. 191

William A. Kirk, On curvature of a metric space at a point . . . . . . . . . . . . 195

G. J. Kurowski, On the convergence of semi-discrete analytic functions . . . . . . . 199

Richard George Laatsch, Extensions of subadditive functions . . . . . . . . . . . 209

V. Marić, On some properties of solutions of $\Delta \psi+A\left(r^{2}\right) X \nabla \psi+C\left(r^{2}\right) \psi=0 \ldots 217$

William H. Mills, Polynomials with minimal value sets . . . . . . . . . . . 225

George James Minty, Jr., On the monotonicity of the gradient of a convex

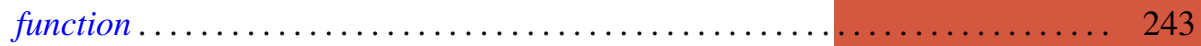

George James Minty, Jr., On the solvability of nonlinear functional equations of 'monotonic' type ................................... 249

J. B. Muskat, On the solvability of $x^{e} \equiv e(\bmod p) \ldots \ldots \ldots \ldots \ldots \ldots \ldots \ldots . \ldots \ldots$

Zeev Nehari, On an inequality of $P . R$. Bessack ................... 261

Raymond Moos Redheffer and Ernst Gabor Straus, Degenerate elliptic

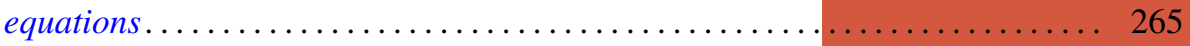

Abraham Robinson, On generalized limits and linear functionals . . . . . . . . . 269

Bernard W. Roos, On a class of singular second order differential equations with a

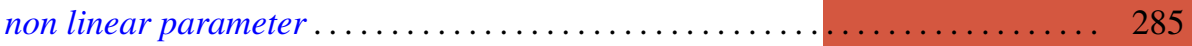

Tôru Saitô, Ordered completely regular semigroups . . . . . . . . . . . . . . . . 295

Edward Silverman, A problem of least area ....................... 309

Robert C. Sine, Spectral decomposition of a class of operators . . . . . . . . . 333

Jonathan Dean Swift, Chains and graphs of Ostrom planes . . . . . . . . . . . 353

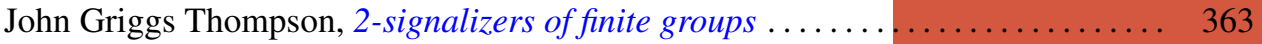

Harold Widom, On the spectrum of a Toeplitz operator . . . . . . . . . . . . . 365 